\title{
Two Sibling Species \\ of the Botrytis cinerea Complex, transposa and vacuma, Are Found in Sympatry on Numerous Host Plants
}

\author{
T. Giraud, D. Fortini, C. Levis, C. Lamarque, P. Leroux, K. LoBuglio, and Y. Brygoo
}

First, second, third, fourth, and seventh authors: INRA, Pathologie Végétale, Route de St Cyr, 78026 Versailles, France; fifth author: INRA, Phytopharmacie, Route de St Cyr, 78026 Versailles, France; and sixth author: Department of Plant Biology, University of California, Berkeley 94720. Accepted for publication 8 July 1999.

\begin{abstract}
Giraud, T., Fortini, D., Levis, C., Lamarque, C., Leroux, P., LoBuglio, K., and Brygoo, Y. 1999. Two sibling species of the Botrytis cinerea complex, transposa and vacuma, are found in sympatry on numerous host plants. Phytopathology 89:967-973.

Strains of Botrytis cinerea (the anamorph of Botryotinia fuckeliana) were collected from 21 different plant species around vineyards in the Champagne region (France). Strains were analyzed using three new polymerase chain reaction (PCR)-restriction fragment length polymorphism (RFLP) markers that were found by SWAPP (sequencing with arbitrary primer pairs), in addition to 15 other markers (PCR-RFLP, transposable elements, and resistance to fungicides). The markers revealed a high degree of genetic diversity and were used to investigate population structure. The two sym-

patric species transposa and vacuma, previously identified on grapes in these vineyards, were also detected on many of the plant species sampled. A new type of strain was also detected, having only the transposable element Boty. We did not detect any differentiation between strains from different organs or locations, but the prevalences of transposa and vacuma were significantly different on the different host plants. Fungicide resistance frequencies were significantly different in transposa and vacuma species. This study confirms that $B$. cinerea is a complex of sibling species and shows that the sibling species occur sympatrically on many host plants. However, the two species seemed to have different pathogenic behaviors. These findings contradict the traditional view of $B$. cinerea as a clonal population without specialization.
\end{abstract}

Botrytis cinerea, the anamorph of Botryotinia fuckeliana, attacks at least 235 plant species in temperate regions (19) and causes grey mold on many economically important crops including vegetables (tomato and cucumber), ornamentals (gerbera and rose), bulbs (onions), and fruits (strawberry and grape). It is also a saprophyte on senescent or already invaded plant materials. Because of the wide range of hosts and tissues that it attacks, and because any strain can infect all plants and organs under laboratory conditions, it had long been thought that $B$. cinerea was not specialized, unlike the other species of this genus. For example, $B$. tulipae is found only on tulips, B. allii on Allium spp., and B. fabae on Leguminosae. However, laboratory experiments may not reflect the natural conditions of infection. In a previous study on grapes in the Champagne region, we showed that $B$. cinerea consists of at least two sympatric sibling species, vacuma and transposa (10). Although both species were present on grapes, there was a dramatic decrease in the percentage of vacuma from June to October. We previously suggested that transposa may be a species more specific to grapes, whereas vacuma may be a migrant species from other hosts. The species transposa was characterized by the presence of two transposable elements, Boty and Flipper, whereas strains of vacuma had neither (10). B. cinerea had been thought to be entirely clonal, but we also showed that it regularly reproduces sexually on grapes in Champagne (10,12).

An understanding of the mode of reproduction and the specialization of phytopathogenic fungi in field conditions is essential for effective disease control. Population dynamics data could be helpful in deciding the best time to apply chemicals, and awareness of

Corresponding author: Y. Brygoo; E-mail address: brygoo@ versailles.inra.fr

Publication no. P-1999-0830-01R

(C) 1999 The American Phytopathological Society the existence of differentiated populations may make it possible to find more efficient methods of control.

The existence of specialization would also be very interesting from a theoretical point of view. It may have played an important role in the speciation of the two sibling sympatric species in $B$. cinerea. There is still no consensus about whether species can arise sympatrically, but it is often argued that specialization on different resources could indeed lead to speciation $(8,9,20,24)$. Sibling species (species indistinguishable on the basis of morphological characters) are often sympatric and specialized on different resources. They may, therefore, have appeared by sympatric speciation due to specialization $(8,9,20,24)$. In fungi, there are many examples of sibling species (2), and most of them are phytopathogens, in which each sibling species uses different resources (hosts, organs, etc.). For example, Puccinia graminis (13,25,27), Erysiphe graminis $(14,15)$, and Fusarium oxysporum (1) all display numerous formae speciales.

To determine whether the two sympatric sibling species transposa and vacuma are specialized, we collected strains from 21 different plants from around grapevines in Champagne. The samples were taken from the same locations as in a previous study on grapes (10). The strains were analyzed using the markers described by Giraud et al. (10): (i) presence of the transposable elements Boty and Flipper; (ii) sensitivity to fungicides (carbendazim, diethofencarb, and vinclozolin, as in the previous study, plus an experimental botryticide, fenhexamid); (iii) polymerase chain reaction (PCR)-restriction fragment length polymorphism (RFLP) markers; and (iv) length of the minisatellite MSB1 (first minisatellite of Botrytis) (11). In addition, we used the SWAPP technique (sequencing with arbitrary primer pairs) (4) to find new PCR-RFLP markers. The objectives of this study were to determine (i) whether the genetic diversity of the complex $B$. cinerea was as great as that on grapes; (ii) whether both transposa and vacuma species were present on other plant species; and (iii) whether the population was structured by the host, location, or organ from which it was collected. 


\section{MATERIALS AND METHODS}

Strains and sampling. A total of 107 field strains of B. cinerea was collected in June 1997 from various plants growing near vineyards in the Champagne region (France). The same three locations (Boursault, Plumecoq, and Trepail) were sampled as in the study on grape strains (10). The strains are listed in Table 1, showing the location, the organ, and the host plant from which each was collected. The strains were not collected inside vine- yards but from a 10-m ring around the vineyard, except in the case of strains from green peas, which were sampled in fields of peas located $100 \mathrm{~m}$ from the vineyards. Some strains were obtained from disease lesions on living plants and others by isolation from asymptomatic tissues. The strains are typed as $\mathrm{S}$ and $\mathrm{P}$, respectively, in Table 1.

The isolates collected on plants were purified by monospore isolation and, therefore, can be referred to as strains. They were preserved in paraffin oil at $16^{\circ} \mathrm{C}$. The fungi were cultured on

TABLE 1. Haplotypes of Botrytis cinerea strains and the location, organ, and host plant from which strains were collected ${ }^{\circ}$

\begin{tabular}{|c|c|c|c|c|c|c|c|c|c|c|c|c|c|c|c|c|c|c|c|c|c|c|}
\hline \multirow[b]{2}{*}{ Isolate } & \multirow[b]{2}{*}{$\mathrm{O}^{\mathrm{p}} \mathrm{F}^{\mathrm{c}}$} & \multirow{2}{*}{\multicolumn{2}{|c|}{$\mathrm{B}^{\mathrm{r}} \begin{array}{c}76^{\mathrm{s}} \\
R s a \mathrm{I}\end{array}$}} & \multicolumn{2}{|c|}{$1^{\mathrm{s}}$} & \multirow{2}{*}{$\mathrm{MSB}^{\mathrm{s}}$} & \multirow{2}{*}{$\begin{array}{l}\mathrm{ADP}^{\mathrm{s}} \\
\text { EcoRI }\end{array}$} & \multicolumn{6}{|c|}{ IGS $^{\mathrm{S}}$} & \multirow{2}{*}{$\begin{array}{c}\text { Nitrate } \\
\text { reductase } \mathrm{e}^{\mathrm{s}} \\
\text { RsaI }\end{array}$} & \multirow{2}{*}{$\mathrm{V}^{\mathrm{t}}$} & Fun & ngicide & & \multirow{2}{*}{\multicolumn{2}{|c|}{$\operatorname{Host}^{\mathrm{y}}$}} & \multirow{2}{*}{\multicolumn{2}{|c|}{$\operatorname{Organ}^{\mathrm{z}}$ Location }} \\
\hline & & & & HaeIII & I HincII & & & $A v a \mathrm{I}$ & $R s a \mathrm{I}$ & BamHI & HindIII & I Hae III & Hin6I & & & $200^{\mathrm{V}}$ & $198^{\mathrm{w}}$ & $\mathrm{F}^{\mathrm{x}}$ & & & & \\
\hline 1148 & S 1 & 11 & 0 & 0 & 1 & 0 & 1 & 1 & 2 & 1 & 1 & 1 & 2 & 1 & 0 & 1 & 1 & 0 & $C$ arvensis & Bindwind & $\mathrm{L}$ & Boursault \\
\hline 1177 & S 1 & 11 & 0 & 0 & & 0 & 1 & 1 & 2 & 1 & 1 & 1 & 2 & 1 & 0 & 1 & 1 & 0 & C. arvensis & Bindwind & $\mathrm{L}$ & Boursault \\
\hline 1178 & S 1 & 11 & & & & 0 & 1 & 1 & 2 & 1 & 1 & 1 & 2 & 1 & 0 & 1 & 1 & 0 & C. arvensis & Bindwind & $\mathrm{L}$ & Boursault \\
\hline 1204 & $\mathrm{P} 1$ & 11 & 0 & 0 & 1 & 0 & 1 & 1 & 1 & 0 & 1 & 1 & 2 & 1 & 0 & 1 & 1 & 0 & C. arvensis & Bindwind & $\mathrm{F}$ & Boursault \\
\hline 1149 & S 1 & 11 & 0 & 0 & 1 & 0 & 1 & 1 & 3 & 0 & 1 & 5 & 2 & 1 & 0 & 1 & 1 & 0 & Rumex acetosella & Sheep sorrel & $\mathrm{L}$ & Boursault \\
\hline 1179 & S 0 & $\begin{array}{ll}0 & 0\end{array}$ & 0 & 0 & 1 & 0 & 1 & 1 & 1 & 1 & 1 & 5 & 2 & 0 & 0 & 1 & 0 & 0 & Rumex acetosella & Sheep sorrel & $\mathrm{L}$ & Boursault \\
\hline 1185 & S 1 & 11 & 0 & 0 & 1 & 0 & 1 & 1 & 3 & 0 & 1 & 1 & 2 & 0 & 0 & 1 & 1 & 0 & Rumex acetosella & Sheep sorrel & $\mathrm{L}$ & Boursault \\
\hline 1152 & S 1 & 11 & 0 & & & 0 & 1 & 1 & 1 & 1 & 1 & 1 & 2 & 1 & 0 & 1 & 1 & 0 & Anagallis arvensis & Scarlet pimpernel & $\mathrm{F}$ & Boursault \\
\hline 1153 & S 1 & 11 & 0 & & & 0 & 1 & 1 & 1 & 1 & 1 & 1 & 2 & 1 & 0 & 1 & 1 & 0 & Anagallis arvensis & Scarlet pimpernel & $\mathrm{F}$ & Boursault \\
\hline 1156 & S 1 & 11 & 0 & & & 2 & 1 & 1 & 1 & 1 & 1 & 1 & 2 & 1 & 0 & 1 & 1 & 0 & Fraxinus sp. & Ash & $\mathrm{L}$ & Boursault \\
\hline 1157 & S 1 & 11 & 0 & & & 2 & 1 & 1 & 1 & 1 & 1 & 1 & 2 & 1 & 0 & 1 & 1 & 0 & Fraxinus sp. & Ash & $\mathrm{L}$ & Boursault \\
\hline 1225 & S 1 & 11 & & & & 1 & & 3 & & 0 & 0 & 9 & 6 & & 0 & 1 & & & Fraxinus sp. & Ash & $\mathrm{L}$ & Boursault \\
\hline 1158 & $\mathrm{P} 1$ & 11 & 1 & & & 0 & 1 & 1 & 1 & 0 & 1 & 1 & 2 & 1 & 0 & 0 & 0 & 0 & G. robertianum & Herb Robert & $\mathrm{F}$ & Boursault \\
\hline 1159 & $\mathrm{P} 1$ & 11 & 0 & 0 & 1 & 0 & 1 & 1 & 1 & 0 & 1 & 1 & 2 & 1 & 0 & 0 & 0 & 0 & G. robertianum & Herb Robert & $\mathrm{F}$ & Boursault \\
\hline 1160 & $\mathrm{P} 1$ & 11 & 0 & 0 & 1 & 0 & 1 & 1 & 1 & 1 & 1 & 1 & 2 & 1 & 1 & 1 & 1 & 0 & G. robertianum & Herb Robert & $\mathrm{F}$ & Boursault \\
\hline 1161 & $\mathrm{P} 1$ & 11 & & 0 & 1 & 2 & 1 & 1 & 1 & 1 & 1 & 1 & 2 & 1 & 0 & 1 & 1 & 0 & G. robertianum & Herb Robert & $\mathrm{F}$ & Boursault \\
\hline 1162 & $\mathrm{P} 1$ & 11 & 0 & 0 & 1 & 0 & 1 & 1 & 1 & 1 & 1 & 1 & 2 & 1 & 1 & 1 & 1 & 0 & G. robertianum & Herb Robert & $\mathrm{F}$ & Boursault \\
\hline 1163 & $\mathrm{P} 1$ & 11 & 0 & & & 0 & 1 & 1 & 1 & 0 & 1 & 1 & 2 & 0 & 0 & 0 & 0 & 0 & G. robertianum & Herb Robert & $\mathrm{F}$ & Boursault \\
\hline 1182 & $\mathrm{P} 1$ & 11 & 0 & 0 & & 0 & 1 & 1 & 1 & 1 & 1 & 1 & 2 & 1 & 0 & 1 & 1 & 0 & G. robertianum & Herb Robert & $\mathrm{F}$ & Boursault \\
\hline 1183 & $\mathrm{P} 1$ & 11 & 0 & 0 & 1 & 1 & 1 & 1 & 1 & 1 & 1 & 0 & 2 & 1 & 1 & 1 & 1 & 0 & G. robertianum & Herb Robert & $\mathrm{F}$ & Boursault \\
\hline 1164 & $\mathrm{P} \quad 0$ & $0 \quad 0$ & 1 & 0 & & 1 & 1 & 0 & 1 & 1 & 1 & 3 & 2 & 0 & 0 & 1 & & & $S$ officinale & Common comphrey & y $F$ & Boursault \\
\hline 1165 & $\mathrm{P} \quad 0$ & $\begin{array}{ll}0 & 0\end{array}$ & 1 & & & 1 & 1 & 0 & 1 & 1 & 1 & 3 & 2 & 0 & 0 & 1 & & & S. officinale & Common comphrey & y $F$ & Boursault \\
\hline 1184 & $\mathrm{P} \quad 0$ & $0 \quad 1$ & 0 & 0 & 1 & 4 & 1 & 1 & 1 & 1 & 1 & 1 & 2 & 0 & 0 & 1 & 0 & 0 & S. officinale & Common comphrey & y $F$ & Boursault \\
\hline 1166 & S 0 & 00 & ) & & & 0 & 1 & 0 & 1 & 1 & 1 & 3 & 2 & 0 & 0 & 1 & & 0 & $P$ acerifolia & Plane tree & L & Boursault \\
\hline 1167 & S 0 & 00 & 1 & 0 & 0 & 0 & 1 & 0 & 1 & 1 & 1 & 3 & 2 & 0 & 0 & 1 & & & P. acerifolia & Plane tree & $\mathrm{L}$ & Boursault \\
\hline 1226 & S 1 & 11 & 0 & & & 2 & 1 & 1 & 1 & 1 & 1 & 0 & 2 & & 0 & 1 & & & P. acerifolia & Plane tree & $\mathrm{L}$ & Boursault \\
\hline 1150 & $\mathrm{P} 0$ & $\begin{array}{ll}0 & 0\end{array}$ & ) & & & 0 & 0 & 0 & 0 & 1 & 1 & 5 & 0 & 0 & 0 & 1 & 1 & 0 & Rubus sp. & Bramble & $\mathrm{P}$ & Boursault \\
\hline 1151 & $\mathrm{P} \quad 0$ & $\begin{array}{ll}0 & 0\end{array}$ & 1 & & & 0 & 0 & 0 & 0 & 1 & 1 & 5 & 0 & 0 & 0 & 1 & 1 & 0 & Rubus sp. & Bramble & $\mathrm{F}$ & Boursault \\
\hline 1168 & $\mathrm{P} \quad 0$ & $\begin{array}{ll}0 & 0\end{array}$ & 0 & 1 & 1 & 5 & 0 & 1 & 0 & 1 & 1 & 2 & 2 & 0 & 0 & 1 & & 0 & Rubus sp. & Bramble & $\mathrm{F}$ & Boursault \\
\hline 1169 & $\mathrm{P} \quad 0$ & $0 \quad 0$ & ) & 2 & 2 & 1 & 1 & 0 & 1 & 1 & 1 & 1 & 2 & 0 & 0 & 1 & 1 & 1 & Rubus sp. & Bramble & $\mathrm{F}$ & Boursault \\
\hline 1170 & $\mathrm{P} 1$ & 11 & 0 & 0 & 1 & 0 & 1 & 1 & 1 & 1 & 1 & 1 & 2 & 1 & 0 & 1 & 1 & 0 & Rubus sp. & Bramble & $\mathrm{F}$ & Boursault \\
\hline 1171 & $\mathrm{P} \quad 0$ & $0 \quad 0$ & 1 & & & 2 & 1 & 0 & 0 & 1 & 1 & 6 & 0 & 1 & 0 & 1 & 0 & 0 & Rubus sp. & Bramble & $\mathrm{F}$ & Boursault \\
\hline 1172 & $\mathrm{P} \quad 0$ & $\begin{array}{ll}0 & 1\end{array}$ & 0 & & & 0 & 1 & 1 & 1 & 0 & 1 & 1 & 2 & 0 & 0 & 1 & 1 & 0 & Rubus sp. & Bramble & $\mathrm{F}$ & Boursault \\
\hline 1173 & $\mathrm{P} \quad 0$ & $\begin{array}{ll}0 & 1\end{array}$ & 0 & & & 0 & 1 & 1 & 1 & 0 & 1 & 1 & 2 & 0 & 0 & 1 & 1 & 0 & Rubus sp. & Bramble & $\mathrm{F}$ & Boursault \\
\hline 1174 & $\mathrm{P} 0$ & $\begin{array}{ll}0 & 0\end{array}$ & 0 & 0 & 1 & 1 & 1 & 1 & 1 & 0 & 0 & 0 & 0 & 0 & 0 & 1 & 0 & 0 & Rubus sp. & Bramble & $\mathrm{F}$ & Boursault \\
\hline 1186 & $\mathrm{P} 0$ & $0 \quad 0$ & 0 & 0 & 1 & 0 & 0 & 0 & 0 & 1 & 1 & 1 & 0 & 0 & 0 & 1 & 1 & 0 & Rubus sp. & Bramble & $\mathrm{F}$ & Boursault \\
\hline 1207 & S 1 & 11 & 0 & 0 & 1 & 0 & 1 & 1 & 1 & 1 & 1 & 1 & 2 & 1 & 0 & 1 & 1 & 0 & Rubus sp. & Bramble & $\mathrm{L}$ & Boursault \\
\hline 1208 & S 1 & 11 & 0 & 0 & 1 & 0 & 1 & 1 & 1 & 1 & 1 & 1 & 2 & 1 & 0 & 1 & 1 & 0 & Rubus sp. & Bramble & $\mathrm{L}$ & Boursault \\
\hline 1209 & S 1 & 11 & 0 & 0 & 1 & 0 & 1 & 1 & 1 & 1 & 1 & 1 & 2 & 1 & 0 & 1 & 1 & 0 & Rubus sp. & Bramble & $\mathrm{L}$ & Boursault \\
\hline 1211 & $\mathrm{P} 1$ & 11 & 0 & 0 & 1 & 0 & 1 & 1 & 1 & 1 & 1 & 1 & 2 & 1 & 0 & 1 & 1 & 0 & Rubus sp. & Bramble & $\mathrm{P}$ & Boursault \\
\hline 1212 & S 1 & 11 & 0 & 0 & 1 & 0 & 1 & 0 & 1 & 1 & 1 & 1 & 2 & 1 & 1 & 1 & 1 & 0 & Rubus sp. & Bramble & $\mathrm{L}$ & Boursault \\
\hline 1213 & $\mathrm{P} 1$ & 11 & 0 & 0 & 1 & 0 & 1 & 1 & 1 & 1 & 1 & 1 & 2 & 1 & 0 & 1 & 1 & 0 & Rubus sp. & Bramble & ST & Boursault \\
\hline 1214 & $\mathrm{P} \quad 1$ & 11 & 0 & 0 & 1 & 0 & 1 & 1 & 1 & 0 & 1 & 1 & 2 & 1 & 0 & 1 & 1 & 0 & Rubus sp. & Bramble & ST & Boursault \\
\hline 1175 & S 1 & 11 & 0 & 0 & 1 & 0 & 1 & 1 & 1 & 1 & 1 & 5 & 2 & 0 & 1 & 1 & 1 & 0 & Tilia sp. & Lime tree & $\mathrm{L}$ & Boursault \\
\hline 1176 & S 1 & 11 & 0 & 0 & & 4 & 1 & 1 & 1 & 1 & 0 & 1 & 2 & 1 & 0 & 1 & 1 & 0 & Tilia sp. & Lime tree & $\mathrm{L}$ & Boursault \\
\hline 1188 & S 1 & 11 & 0 & 0 & 1 & 0 & 1 & 1 & 1 & 1 & 1 & 1 & 2 & 1 & 0 & 1 & 1 & 0 & Tilia sp. & Lime tree & & Boursault \\
\hline
\end{tabular}

(continued on the next page)

o Blanks within the table indicate that the data was not determined.

$\mathrm{p} \mathrm{O}=$ Obtained. $\mathrm{S}$ indicates that the strain was obtained from disease lesions on living plants, and $\mathrm{P}$ indicates that the strain was obtained from asymptomatic tissues.

${ }^{\mathrm{q}} \mathrm{F}=$ Flipper. The absence of Flipper is coded by 0 and its presence by 1 .

${ }^{\mathrm{r}} \mathrm{B}=$ Boty. The absence of Boty is coded by 0 and its presence by 1 .

$s$ Each allele of polymerase chain reaction-restriction fragment length polymorphism markers is coded by a different number.

${ }^{\mathrm{t}} \mathrm{V}=$ Vinclozolin. The sensitivity to vinclozolin (ImiS) is coded by 0 and the resistance (ImiR) by 1 .

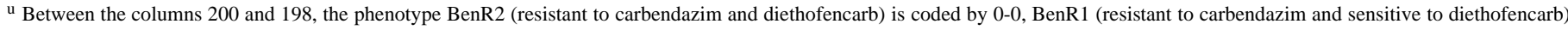
by 1-1, and BenS (sensitive to carbendazim and resistant to diethofencarb) by 1-0.

v $200=$ Codon 200. At the codon 200 of the $\beta$-tubulin, 1 is for the presence of a phenylalanine and 0 for the presence of tyrosine.

${ }^{\mathrm{w}} 198=$ Codon 198. At the codon 198 of the $\beta$-tubulin, 1 is for the presence of an alanine and 0 for the presence of glutamic acid.

${ }^{\mathrm{x}} \mathrm{F}=$ Fenhexamid. The sensitivity to fenhexamid (HydS) is coded by 0 and the resistance (HydR) by 1 .

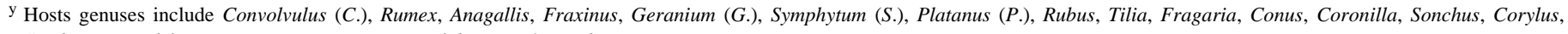
Sambuscus, Trifolium, Pisum, Brassica, Rosa, Epilobium, and Reseda.

${ }^{\mathrm{z}} \mathrm{L}=$ Leaf, $\mathrm{F}=$ flower, $\mathrm{P}=$ petal, $\mathrm{ST}=$ stamen, $\mathrm{FR}=$ fruit, $\mathrm{PO}=$ pod, and $\mathrm{S}=$ sepal. 
plates containing $20 \mathrm{ml}$ of nutrient yeast medium ( $20 \mathrm{~g}$ of malt per liter, $20 \mathrm{~g}$ of agar per liter, and $2 \mathrm{~g}$ of yeast extract per liter) at $25^{\circ} \mathrm{C}$.

Fungicide sensitivity assays. Conidia were collected in sterile water from 2 -week-old agar slant cultures. The resulting spore suspension $\left(2 \times 10^{5}\right.$ conidia per $\left.\mathrm{ml}\right)$ was used to inoculate a glucose agar medium containing fungicides (17). Fungicides from four chemical groups were tested: benzimidazoles (carbendazim), phenylcarbamates (diethofencarb), dicarboximides (vinclozolin), and hydroxyanilides (fenhexamid). The discriminatory concentrations were 1 and
$10 \mathrm{mg} /$ liter for carbendazim, $10 \mathrm{mg} /$ liter for diethofencarb, $5 \mathrm{mg} / \mathrm{liter}$ for vinclozolin, and $0.4 \mathrm{mg} / \mathrm{liter}$ for fenhexamid (Table 2). The response of the various strains was recorded after incubation for 1 or 2 days.

DNA extraction and molecular markers. The mycelium was cultured as described by Diolez et al. (6). Genomic DNA was isolated by the method of Möller et al. (21). The primers, the amplification, and the digestion conditions used for the PCR-RFLP markers were the same as those described by Giraud et al. (10). The length

\begin{tabular}{|c|c|c|c|c|c|c|c|c|c|c|c|c|c|c|c|c|c|c|c|c|c|c|c|}
\hline \multirow[b]{2}{*}{ Isolate } & \multirow{2}{*}{\multicolumn{3}{|c|}{$\mathrm{O}^{\mathrm{p}} \mathrm{F}^{\mathrm{q}} \mathrm{B}^{\mathrm{r}}$}} & \multirow{2}{*}{$\begin{array}{c}76^{\mathrm{s}} \\
R s a \mathrm{I}\end{array}$} & \multicolumn{2}{|c|}{$1^{\mathrm{s}}$} & \multirow{2}{*}{$\mathrm{MSB}_{1}^{\mathrm{s}}$} & \multirow{2}{*}{$\begin{array}{l}\mathrm{ADP}^{\mathrm{s}} \\
\text { EcoRI }\end{array}$} & & & IG & $\mathrm{iS}^{\mathrm{s}}$ & & & $\begin{array}{c}\text { Nitrate } \\
\text { reductase }^{\mathrm{s}}\end{array}$ & & Fun & $\begin{array}{l}\text { ngicide: } \\
\mathrm{en}^{\mathrm{u}} \\
\end{array}$ & & & & & \\
\hline & & & & & HaeIII & HincII & & & $A v a \mathrm{I}$ & $R s a \mathrm{I}$ & BamHI & HindIII & I HaeIII & Hin6I & $R s a \mathrm{I}$ & $\mathrm{V}^{\mathrm{t}}$ & $200^{\mathrm{V}}$ & $198^{\mathrm{w}}$ & $\mathrm{F}^{\mathrm{x}}$ & Hos & $\mathrm{st}^{\mathrm{y}}$ & Organ & ${ }_{1}^{\mathrm{z}}$ Location \\
\hline 1189 & $\mathrm{P}$ & 0 & 0 & 0 & 2 & 2 & 1 & 0 & 0 & 1 & 1 & 1 & 3 & 2 & 0 & 0 & 1 & 1 & 1 & Tilia sp. & Lime tree & $\mathrm{F}$ & Boursault \\
\hline 1190 & $\mathrm{P}$ & 0 & 0 & & & & & & 0 & 4 & 2 & 0 & 1 & 4 & & & & & & Tilia sp. & Lime tree & $\mathrm{F}$ & Boursault \\
\hline 1180 & $S$ & 0 & 0 & & & & & & 2 & 2 & 0 & 0 & 7 & 3 & & & & & & Fragaria sp. & Strawberry & FR & Boursault \\
\hline 1192 & $\mathrm{P}$ & 1 & 1 & 0 & 0 & & 1 & 1 & 1 & 1 & 1 & 1 & 1 & 2 & 0 & 0 & 1 & 0 & 0 & Conus mas & Cornel tree & $\mathrm{F}$ & Boursault \\
\hline 1193 & $P$ & 1 & 1 & 0 & & & 1 & 1 & 1 & 1 & 1 & 1 & 1 & 2 & 1 & 0 & 1 & 0 & 0 & Conus mas & Cornel tree & $\mathrm{F}$ & Boursault \\
\hline 1194 & $\mathrm{P}$ & 1 & 1 & 0 & 2 & 2 & 0 & 1 & 1 & 1 & 1 & 1 & 1 & 2 & 0 & 1 & 1 & 1 & 0 & Conus mas & Cornel tree & $\mathrm{F}$ & Boursault \\
\hline 1195 & $\mathrm{P}$ & 1 & 1 & & & & 1 & 1 & 1 & 1 & 1 & 1 & 1 & 2 & 1 & 1 & 1 & 1 & 0 & Conus mas & Cornel tree & $\mathrm{F}$ & Boursault \\
\hline 1197 & $\mathrm{P}$ & 1 & 1 & 0 & & & 1 & 1 & 1 & 1 & 0 & 1 & 1 & 2 & 1 & 0 & 1 & 1 & 0 & Coronilla coronata & Coronilla & $\mathrm{F}$ & Boursault \\
\hline 1198 & & 0 & 0 & & & & 1 & 1 & 1 & 1 & 1 & 1 & & 2 & & 0 & 1 & 1 & 1 & Sonchus arvensis & Milkweed & $\mathrm{F}$ & Boursault \\
\hline 1199 & & 0 & 0 & 1 & & & 1 & 1 & 0 & 1 & 1 & 1 & 3 & 2 & 0 & 0 & 1 & 1 & 1 & Sonchus arvensis & Milkweed & $\mathrm{F}$ & Boursault \\
\hline 1200 & & 1 & 1 & 0 & 0 & 1 & 2 & 1 & 1 & 1 & 1 & 1 & 0 & 2 & 0 & 0 & 1 & 1 & 0 & Sonchus arvensis & Milkweed & $\mathrm{F}$ & Boursault \\
\hline 1202 & & 1 & 1 & 0 & 0 & 1 & 2 & 1 & 1 & 1 & 1 & 1 & 0 & 2 & 0 & 0 & 1 & 1 & 0 & Sonchus arvensis & Milkweed & $\mathrm{F}$ & Boursault \\
\hline 1203 & & 1 & 1 & 0 & 0 & 1 & 2 & 1 & 1 & 1 & 1 & 1 & 0 & 2 & 1 & 0 & 1 & 1 & 0 & Sonchus arvensis & Milkweed & $\mathrm{F}$ & Boursault \\
\hline 1205 & $S$ & 1 & 1 & 0 & 0 & 1 & 2 & 1 & 1 & 1 & 1 & 1 & 1 & 2 & 1 & 0 & 1 & 1 & 0 & Corylus avellana & Bazel tree & $\mathrm{F}$ & Boursault \\
\hline 1206 & $S$ & 1 & 1 & & & & 2 & 1 & 1 & 1 & 1 & 1 & 1 & 2 & 1 & 0 & 1 & 1 & 0 & Corylus avellana & Bazel tree & $\mathrm{F}$ & Boursault \\
\hline 1215 & S & 0 & 0 & 0 & 1 & & 0 & 0 & 0 & 0 & 1 & 1 & 3 & 0 & 1 & 0 & 1 & 1 & 0 & Sambuscus nigra & Common elder & $\mathrm{L}$ & Boursault \\
\hline 1217 & $\mathrm{~S}$ & 1 & 1 & 0 & 0 & & 0 & 1 & 1 & 1 & 1 & 1 & 0 & 2 & 0 & 0 & 1 & 1 & 0 & Sambuscus nigra & Common elder & $\mathrm{L}$ & Boursault \\
\hline 1218 & $\mathrm{~S}$ & 1 & 1 & 0 & 0 & & 0 & 1 & 1 & 1 & 1 & 1 & 1 & 2 & 1 & 0 & 1 & 1 & 0 & Sambuscus nigra & Common elder & $\mathrm{L}$ & Boursault \\
\hline 1219 & $\mathrm{~S}$ & 1 & 1 & & & & 0 & 1 & 1 & 1 & 1 & 1 & 1 & 2 & 1 & 0 & 1 & 1 & 0 & Sambuscus nigra & Common elder & $\mathrm{L}$ & Boursault \\
\hline 1220 & $\mathrm{~S}$ & 1 & 1 & 0 & 1 & & 0 & 1 & 1 & 1 & 1 & 1 & 1 & 2 & 0 & 0 & 1 & 1 & 0 & Sambuscus nigra & Common elder & $\mathrm{L}$ & Boursault \\
\hline 1221 & $\mathrm{P}$ & 0 & 0 & 1 & 2 & 2 & 0 & 1 & 0 & 1 & 1 & 1 & 3 & 2 & 0 & 0 & 1 & 1 & 1 & Trifolium repens & White clover & $\mathrm{F}$ & Boursault \\
\hline 1222 & $P$ & 0 & 0 & 1 & 0 & & 1 & 1 & 0 & 1 & 1 & 1 & 3 & 2 & 0 & 0 & 1 & 1 & 1 & Trifolium repens & White clover & $\mathrm{F}$ & Boursault \\
\hline 1223 & $P$ & 0 & 0 & 1 & 2 & 2 & 1 & 1 & 0 & 1 & 1 & 1 & 6 & 2 & 0 & 0 & 1 & & & Trifolium repens & White clover & $\mathrm{F}$ & Boursault \\
\hline 1272 & $\mathrm{P}$ & 1 & 1 & 0 & & & 0 & & 1 & 1 & 0 & 1 & 1 & 2 & & 0 & 1 & 1 & 0 & Trifolium repens & White clover & $\mathrm{L}$ & Plumecoq \\
\hline 1229 & $\mathrm{P}$ & 0 & 0 & 1 & & & 1 & 1 & & & 0 & & & & 1 & 0 & 1 & 1 & 1 & Pisum sativum & Green pea & $\mathrm{F}$ & Trépail \\
\hline 1230 & $\mathrm{P}$ & 0 & 0 & 1 & 2 & 2 & 1 & 1 & 0 & 1 & 0 & 1 & 3 & 2 & & 0 & 1 & & & Pisum sativum & Green pea & $\mathrm{F}$ & Trépail \\
\hline 1231 & $P$ & 0 & 0 & & & & 1 & 1 & 0 & 1 & 1 & 1 & 3 & 2 & 0 & 0 & 1 & & & Pisum sativum & Green pea & $\mathrm{F}$ & Trépail \\
\hline 1232 & $\mathrm{P}$ & 1 & 1 & 0 & 0 & 1 & 2 & 1 & 1 & 1 & 1 & 1 & 0 & 2 & & 1 & 1 & 1 & 0 & Pisum sativum & Green pea & $\mathrm{F}$ & Trépail \\
\hline 1233 & $\mathrm{P}$ & 0 & 1 & 0 & 0 & 1 & 1 & 1 & 1 & 1 & 0 & 1 & & 2 & & 0 & 1 & 1 & 0 & Pisum sativum & Green pea & $\mathrm{F}$ & Trépail \\
\hline 1258 & $\mathrm{P}$ & 0 & 0 & 1 & & & 0 & 1 & 0 & 1 & 1 & 1 & & & 0 & 0 & 1 & 1 & 1 & Pisum sativum & Green pea & $\mathrm{F}$ & Plumecoq \\
\hline 1259 & $\mathrm{P}$ & 0 & 0 & 0 & & & 0 & 0 & 1 & 0 & 1 & 1 & 5 & 1 & 1 & 0 & 1 & 1 & 0 & Pisum sativum & Green pea & $\mathrm{F}$ & Plumecoq \\
\hline 1260 & $\mathrm{P}$ & 0 & 0 & 0 & 0 & 1 & 0 & 0 & 1 & 0 & 1 & 1 & 5 & 1 & 0 & 0 & 1 & 1 & 0 & Pisum sativum & Green pea & $\mathrm{F}$ & Plumecoq \\
\hline 1261 & $\mathrm{P}$ & 0 & 0 & 0 & 0 & 1 & 0 & 0 & 1 & 0 & 1 & 1 & 5 & 2 & 0 & 0 & 1 & 1 & 0 & Pisum sativum & Green pea & $\mathrm{F}$ & Plumecoq \\
\hline 1262 & $\mathrm{P}$ & 0 & 0 & 1 & 1 & 1 & 1 & 1 & 0 & 1 & 1 & 1 & & 2 & 0 & 0 & 1 & 1 & 1 & Pisum sativum & Green pea & PO & Plumecoq \\
\hline 1263 & $\mathrm{P}$ & 0 & 0 & 1 & & & 1 & 1 & 0 & 1 & 1 & 1 & & 2 & 0 & 0 & 1 & 1 & 1 & Pisum sativum & Green pea & $\mathrm{PO}$ & Plumecoq \\
\hline 1264 & $P$ & 0 & 0 & 1 & & & 1 & 1 & & & & 0 & & & 0 & 0 & 1 & 1 & 1 & Pisum sativum & Green pea & PO & Plumecoq \\
\hline 1268 & $\mathrm{P}$ & 0 & 0 & 1 & & & 1 & 1 & & & & 0 & & 2 & 0 & 0 & 1 & 1 & 1 & Pisum sativum & Green pea & $\mathrm{PO}$ & Plumecoq \\
\hline 1236 & $\mathrm{P}$ & 0 & 0 & 1 & 0 & 1 & 0 & 1 & 1 & 1 & 1 & 1 & 1 & 2 & 0 & 0 & 1 & 0 & 0 & Brassica napus & Rapeseed & $\mathrm{F}$ & Trépail \\
\hline 1237 & $\mathrm{P}$ & 0 & 0 & 0 & 0 & 1 & 0 & 1 & 1 & 1 & 1 & 1 & 1 & 2 & 0 & 0 & 1 & 0 & 0 & Brassica napus & Rapeseed & $\mathrm{F}$ & Trépail \\
\hline 1238 & $P$ & 0 & 0 & 0 & 0 & 1 & 0 & 1 & 1 & 1 & 0 & 1 & 1 & 2 & 0 & 0 & 1 & 0 & 0 & Brassica napus & Rapeseed & FR & Trépail \\
\hline 1239 & $P$ & 0 & 0 & 0 & 0 & 1 & 0 & 1 & 1 & 1 & 0 & 1 & 1 & 2 & & 0 & 1 & 0 & 0 & Brassica napus & Rapeseed & FR & Trépail \\
\hline 1240 & $\mathrm{~S}$ & 1 & 1 & 1 & 0 & & 0 & 1 & 0 & 1 & 0 & 1 & 3 & 2 & 0 & 0 & 1 & 1 & 1 & Rosa canina & Wild rose & $\mathrm{P}$ & Plumecoq \\
\hline 1241 & $\mathrm{~S}$ & 1 & 1 & 0 & 0 & 1 & 2 & 1 & 0 & 0 & 1 & 1 & 3 & & 0 & 0 & 1 & 1 & 0 & Rosa canina & Wild rose & $\mathrm{S}$ & Plumecoq \\
\hline 1242 & $\mathrm{~S}$ & 0 & 0 & 1 & 2 & 2 & 1 & 1 & 0 & 1 & 1 & 1 & 3 & 2 & 0 & 0 & 1 & 0 & 1 & Rosa canina & Wild rose & $\mathrm{S}$ & Plumecoq \\
\hline 1243 & $\mathrm{~S}$ & 0 & 0 & 1 & 2 & 2 & 1 & 1 & 0 & 1 & 0 & 1 & & 2 & 0 & 0 & 1 & 0 & 1 & Rosa canina & Wild rose & $\mathrm{S}$ & Plumecoq \\
\hline 1244 & $\mathrm{~S}$ & 0 & 0 & 1 & & & 1 & 1 & 0 & 1 & 0 & 1 & & 2 & 0 & 0 & 1 & 0 & 1 & Rosa canina & Wild rose & $S$ & Plumecoq \\
\hline 1245 & $\mathrm{~S}$ & 0 & 0 & 1 & 2 & 2 & 1 & 1 & 0 & 1 & & 1 & 3 & & 1 & 0 & 1 & 0 & 1 & Rosa canina & Wild rose & $S$ & Plumecoq \\
\hline 1246 & S & 0 & 0 & 1 & 2 & 2 & 1 & 1 & 0 & & 0 & 1 & & 2 & 0 & 0 & 1 & 0 & 1 & Rosa canina & Wild rose & $\mathrm{S}$ & Plumecoq \\
\hline 1247 & $\mathrm{~S}$ & 0 & 0 & 0 & & & 1 & 1 & 0 & 1 & 1 & 1 & 3 & 2 & 0 & 0 & 1 & 0 & 1 & Rosa canina & Wild rose & $\mathrm{S}$ & Plumecoq \\
\hline 1248 & $\mathrm{~S}$ & 0 & 0 & 0 & & & 1 & 1 & 0 & & 1 & 1 & & 2 & 0 & 0 & 1 & 0 & 1 & Rosa canina & Wild rose & $\mathrm{S}$ & Plumecoq \\
\hline 1249 & $\mathrm{~S}$ & 1 & 1 & & & & 4 & & 2 & 4 & 1 & 0 & 8 & 5 & & 0 & 1 & 0 & & Rosa canina & Wild rose & $\mathrm{S}$ & Plumecoq \\
\hline 1250 & $\mathrm{~S}$ & 0 & 1 & & & & 4 & & 2 & 4 & 1 & 0 & 8 & 5 & & 0 & 1 & 0 & & Rosa canina & Wild rose & $\mathrm{L}$ & Plumecoq \\
\hline 1251 & $\mathrm{~S}$ & 0 & 1 & & 1 & 1 & 4 & 1 & 2 & 4 & 1 & 0 & 8 & 5 & & 0 & 1 & 0 & & Rosa canina & Wild rose & $\mathrm{L}$ & Plumecoq \\
\hline 1252 & $\mathrm{~S}$ & 0 & 0 & 1 & & & 1 & 1 & 0 & 1 & 1 & 1 & & 2 & 0 & 0 & 1 & 1 & 1 & Rosa canina & Wild rose & $\mathrm{L}$ & Plumecoq \\
\hline 1253 & $P$ & 0 & 0 & 0 & & & 2 & & 0 & 1 & 1 & 1 & & & 0 & 0 & 1 & 0 & 0 & Rosa canina & Wild rose & ST & Plumecoq \\
\hline 1254 & $\mathrm{P}$ & 1 & 1 & 0 & 0 & 1 & 4 & 1 & 1 & 1 & 1 & 1 & 1 & 2 & 1 & 1 & 1 & 1 & 0 & Rosa canina & Wild rose & ST & Plumecoq \\
\hline 1256 & $\mathrm{~S}$ & 0 & 0 & 1 & & & 1 & 1 & 0 & 1 & 1 & 1 & 3 & 2 & 0 & 0 & 1 & 1 & 1 & Epilobium sp. & Willow herb & $\mathrm{L}$ & Plumecoq \\
\hline 1257 & S & 0 & 0 & 0 & 0 & 1 & 0 & 0 & 0 & & & & & & & 0 & 1 & 1 & 0 & Epilobium sp. & Willow herb & $\mathrm{L}$ & Plumecoq \\
\hline 1269 & $P$ & 0 & 1 & 0 & & & & 1 & & & & & & 2 & & 0 & 1 & 0 & 0 & Reseda lutea & Yellow weed & $\mathrm{F}$ & Plumecoq \\
\hline 1270 & $P$ & 0 & 1 & 0 & 0 & 1 & 5 & 1 & 1 & 1 & 0 & 1 & 1 & 2 & & 0 & 1 & 0 & 0 & Reseda lutea & Yellow weed & $\mathrm{F}$ & Plumecoq \\
\hline 1271 & $\mathrm{P}$ & 1 & 1 & 0 & 0 & 1 & 0 & 1 & 1 & 1 & 0 & 0 & 3 & 2 & 1 & 0 & 1 & 0 & 0 & Reseda lutea & Yellow weed & $\mathrm{F}$ & Plumecoq \\
\hline
\end{tabular}


of the minisatellite MSB1 was scored by PCR as described by Giraud et al. (11). The DNA fragments were then separated by electrophoresis in $1.5 \%$ agarose gels and in $1 \times$ Tris-borate-EDTA buffer.

Dot blots were performed as described by Giraud et al. (10) to detect the transposable elements Boty and Flipper. The presence or absence of Flipper was checked by PCR for some strains. The primers used were F300 (5'GCACAAAACCTACAGAAGA3') and F1500 (5'ATTCGTTTCTTGGACTGTA3') (18). Boty is a 6-kilobase gypsy-like retrotransposon (accession numbers X81790 and X81791) (6). Flipper is a 1,842-base pair (bp) class II element (accession number U74294) (18). Both were isolated from $B$. cinerea.

For the SWAPP technique, random amplification of polymorphic DNA (RAPD), and single-strand conformational polymorphism (SSCP) were done as described by Burt et al. (5). For sequencing, PCR products were purified using the Jetsorb gel extraction kit (Bioprobe Systems, Montreuil-sous-Bois, France) and sequenced using the ABI PRISM Dye Terminator Cycle Sequencing Ready Reaction Kit with AmpliTaq DNA Polymerase (Perkin-Elmer, Branchburg, NJ) and the ABI PRISM 310 Genetic Analyzer (Perkin-Elmer).

Data analysis. Allele frequencies and genetic differentiation were calculated using the GENEPOP program (23). The null hypothesis tested for genetic differentiation was $\mathrm{H}_{0}$ (the allelic distribution is independent across populations). GENEPOP performs Fisher's exact tests using a Markov chain. The gene diversity $\left(\mathrm{H}_{\mathrm{s}}\right)$ at each locus was calculated by the Nei's method (22): $\mathrm{H}_{\mathrm{s}}=1-$ $\sum p_{i}{ }^{2}$, in which $p_{i}$ is the frequency of the $i$ th allele. The mean gene diversity was the mean of $\mathrm{H}_{\mathrm{s}}$ over loci.

\section{RESULTS}

SWAPP. The principle of the method is to amplify random segments of DNA using RAPDs with pairs of primers and then to select bands that are in all samples of interest but do not appear when either primer is used alone. These bands are then scored by SSCP, a technique that detects differences in conformation of single strands of DNA by migration in a denaturing gel. Polymorphic bands are then sequenced. We tried 31 primers in 42 arbitrary pairs

TABLE 2. Botrytis cinerea strains classified according to response of phenotypes to different fungicides

\begin{tabular}{lccccc}
\hline & \multicolumn{2}{c}{ Carbendazim } & Diethofencarb & Vinclozolin & Fenhexamid \\
\cline { 2 - 4 } Phenotypes & $1^{\mathrm{x}}$ & 10 & 10 & 5 & \\
\hline Wild types $^{\mathrm{y}}$ & $-^{\mathrm{z}}$ & - & + & - & - \\
BenR1 & + & + & - & - & - \\
BenR2 & + & - & + & - & - \\
ImiR & - & - & + & + & - \\
HydR & - & - & + & - & + \\
\hline
\end{tabular}

${ }^{\mathrm{x}}$ Fungicide concentrations are given in $\mathrm{mg} / \mathrm{liter}$.

${ }^{y}$ Wild types include BenS, ImiS, and HydS.

${ }^{z}-=$ Absence of germ tubes or presence of short germ tubes. $+=$ Presence of long germ tubes. on 10 strains. Six primer pairs revealed eight polymorphic bands by SSCP (Table 3) that were then sequenced. None of the sequences showed a similarity with sequences present in GenBank. The sequences revealed polymorphic restriction sites (Table 3), and three enzymes were used in extensive population studies: HaeII, $H i n c I I$, and RsaI. The enzymes HaeII and HincII had polymorphic restriction sites in the band amplified by the pair of primers no. 1 (primers ITS4 and ITS5) (Table 4), and RsaI had polymorphic restriction sites in the band amplified by the pair of primers no. 76 (primers L76-5' and L76-3'). We designed specific primers for these two bands (76U and 76L, 1BU and 1BL) (Table 4) and screened all the strains for polymorphism by digesting band 1 with $R s a \mathrm{I}$ and band 76 with HaeII and HincII. With marker 76, allele 0 (not cut by RsaI) was found in $71 \%$ of the strains and allele 1 (cut by $R s a \mathrm{I})$ in $28.6 \%$. With marker 1 , a larger band (830 bp) was always coamplified with the 500-bp band. The 830-bp band exhibited a polymorphic amplification length: $15.4 \%$ of the strains displayed a higher and fainter band at 950 bp (Fig. 1, alleles 2). This 950-bp band is presumably homologous to the 830-bp band, since they never occurred together. After HaeIII digestion, allele 0 (not cut) was found in $76.9 \%$ of the strains and allele 1 (cut) in $7.7 \%$. After HincII digestion, allele 0 (not cut) was found in $1.8 \%$ of the strains and allele 1 (cut) in $79.6 \%$. The alleles are shown in Figure 1. These are good markers for population studies, since no allele is ever too prevalent.

Population analysis. The 107 strains analyzed are listed in Table 1. They were collected from 21 different plants around grapevines in Champagne, from the same locations as in the study on strains from grapes (10), and they were scored for 18 markers. Strains were scored for MSB1 length polymorphism (11) and for 11 PCRRFLP markers by amplifying two coding sequences (nitrate reductase and ADP/ATP translocase), one noncoding repeated sequence (nontranscribed ribosomal intergenic spacer [IGS]), and the two anonymous SWAPP sequences (1 and 76). The IGS was digested by six enzymes (AvaI, RsaI, BamHI, HindIII, HaeIII, and Hin6I), the ADP/ATP translocase was digested by EcoRI, and the nitrate reductase was digested by $R s a \mathrm{I}$. Restriction patterns were described by Giraud et al. (10). The band 1 obtained by SWAPP was digested by HaeIII and HincII, and the band 76 was digested by RsaI. Restriction patterns are shown in Figure 1. Different digestions of the IGS are considered to be different markers, because recombination can occur between these sites (10). The 107 strains were then scored for the presence of two transposable elements (Boty and Flipper) by dot blots.

The strains were also tested for their resistance to four fungicides (carbendazim, diethofencarb, vinclozolin, and fenhexamid). Several phenotypes were identified on the basis of in vitro effects of these fungicides on the various strains (Table 2). Three phenotypes were distinguished by their response to antimicrotubule fungicides: BenS (sensitive to carbendazim and resistant to diethofencarb), BenR1 (resistant to carbendazim and sensitive to diethofencarb), and BenR2 (resistant to both carbendazim and diethofencarb). Carbendazim-resistant strains differ from sensitive ones by two pairs of mutations in the beta-tubulin gene (28). Alanine replaces glu-

TABLE 3. Polymorphism detected by SWAPP (sequencing with arbitrary primer pairs)

\begin{tabular}{lccccc}
\hline Primers & Band & $\begin{array}{c}\text { Strains with polymorphic } \\
\text { band by SSCP }\end{array}$ & $\begin{array}{c}\text { No. of polymorphic base pairs } \\
\text { detected by sequencing }\end{array}$ & $\begin{array}{c}\text { No. of polymorphic restriction sites } \\
\text { determined by sequencing }\end{array}$ & \multicolumn{1}{c}{ Enzymes } \\
\hline ITS4/ITS5 & & $349,111,1081,187$ & 8 & 5 & MnlI, MseI, HincII, HaeIII, NlaIII \\
ITS4/NS24 & & $111,1081,1069$ & 2 & 0 & MboII \\
TW14/Trpc1 & A & 169,158 & 1 & 0 & FokI \\
TW14/Trpc1 & B & 169,158 & 0 & 1 & ItaI \\
MS2/NS4 & & 158,846 & 2 & 1 & \\
NS7/ML3.5 & A & 1069,187 & 2 & 0 & RsaI, FokI, NsiI \\
NS7/ML3.5 & B & 1069,187 & 0 & 3 & \\
L76-5'/L76-3' & & 1069,187 & 5 & 0 & \\
\hline
\end{tabular}

${ }^{\mathrm{z}}$ Primers that gave one or two polymorphic bands by single-strand conformational polymorphism (SSCP), polymorphism detected by sequencing these bands, and polymorphism of restriction. 
tamic acid (codon 198) in BenR1, whereas tyrosine replaces phenylalanine (codon 200) in BenR2. The field strains were either sensitive (ImiS) or moderately resistant (ImiR) to vinclozolin. This resistance is encoded by a single gene (7), the function of which remains to be investigated. The in vitro tests conducted with the experimental hydroxyanilide botryticide, fenhexamid, distinguished sensitive (HydS) and resistant (HydR) strains. The genetic basis of this resistance is not known, but according to Suty et al. (26), it seems to be related to increased detoxification of this hydroxyanilide. All the fenhexamid-resistant strains, but none of the sensitive ones, exhibited increased sensitivity to fenpropimorph, as did the strains collected from grapes (16).

Population structure. The haplotypes of the 107 strains are shown in Table 1 . We found, as in our previous study (10), that almost all strains either had both transposable elements or neither of them. That means that the two distinct species of B. cinerea, transposa and vacuma, are also found on hosts other than grapes. The percentage of vacuma is $45 \%$, which is the same as that found in the strains collected from grapes in June 1994 and June 1995 (10). The sibling species transposa and vacuma were found to be sympatric on 11 out of the 16 plants from which more than two strains were collected. However, we also detected eight strains that had only the transposable element Boty. Such strains had not been detected before in France, but some were found in Chile (K. LoBuglio, unpublished data).

TABLE 4. Sequences of primers used for SWAPP (sequencing with arbitrary primer pairs)

\begin{tabular}{ll}
\hline Primer & \multicolumn{1}{c}{ Sequence $\left(5^{\prime}-3^{\prime}\right)$} \\
\hline NS4 & CTTCCGTCAATTCCTTTAAG \\
NS7 & GAGGCAATAACAGGTCTGTGATGC \\
NS24 & AAACCTTGTTACGACTTTA \\
MS2 & GCGGATTATCGAATTAAATAAC \\
L76-5 & CTGAGAAACCACTGCGTAG \\
L76-3 & CCATGAACAAATATGGC \\
ITS4 & TCCTCCGCTTATTGATATGC \\
ITS5 & GGAAGTAAAAGTCGGTAACAAGG \\
TW14 & GCTATCCTGAGGGAAACTTC \\
ML3.5 & GAGAATGCTGACATGAGTAAC \\
Trpc1 & GTGCCAGTGTCATCTCTGTGCT \\
76U & AATTGGAATTTGTGTTGGTC \\
76L & ATTGCTATCCTGAGGGAAAC \\
1BU & ACAAAAGGGGAGGGAGGTAT \\
1BL & TCGAGGGAAAGAAAGAAAGG \\
\hline
\end{tabular}

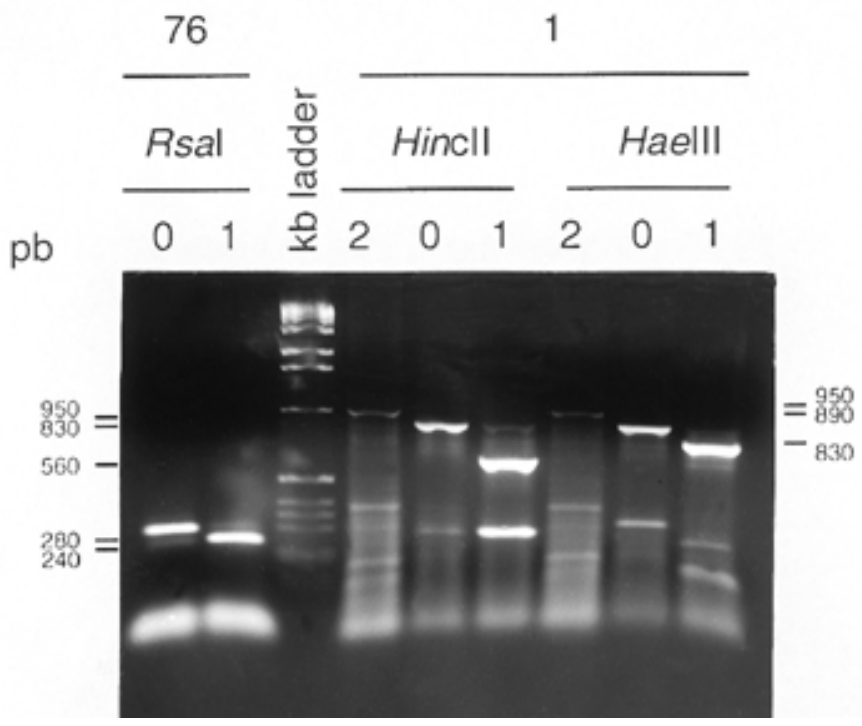

Fig. 1. Restriction patterns of the alleles of the three polymerase chain reactionrestriction fragment length polymorphism markers obtained for Botrytis cinerea by SWAPP (sequencing with arbitrary primer pairs).
To determine whether the population was structured, we subdivided it on the basis of organ, location, and host plant, and calculated the allele frequencies for each marker in each subgroup. We then performed a statistical test to see whether allele frequencies were significantly different in the subgroups. The plants from which less than four strains were collected were excluded from this analysis. The differences were considered significant if the probability $(P)$ that they occurred by chance was less than $5 \%$. There were no significant differences between the groups from different locations or between the groups from different organs. However, significant differences in allele frequencies were found for 11 out of the 18 markers among strains collected from different host plants (Table 5). Some plant species carried only transposa or only vacuma, and 11 plants carried both transposa and vacuma. But the frequencies of strains having Boty and Flipper were significantly different between the different plant hosts (Table 5). Thus, even though transposa and vacuma were sometimes found together on some plant species, their relative prevalences were significantly different. For example, vacuma is prevalent on green peas, whereas transposa is prevalent on bramble and herb Robert (Table 6). As transposa and vacuma have different

TABLE 5. Test of allelic differences among the strains of Botrytis cinerea collected from different plant hosts

\begin{tabular}{lc}
\hline Locus & $P^{\mathrm{z}}$ \\
\hline Flipper & $0.00004^{*}$ \\
Boty & $0.00004^{*}$ \\
76 RsaI & $0.03354^{*}$ \\
1 HaeIII & 0.35576 \\
1 HincII & $0.01452^{*}$ \\
ATP synthase & $<0.000001^{*}$ \\
ADP-ATP translocase/EcoRI & 0.60276 \\
IGS/AvaI & $<0.000001^{*}$ \\
IGS/RsaI & $0.002306^{*}$ \\
IGS/BamHI & 0.31006 \\
IGS/HindIII & 0.26588 \\
IGS/HaeIII & $<0.000001^{*}$ \\
IGS/Hin6I & 0.11486 \\
Nitrate reductase/RsaI & $0.00040^{*}$ \\
Vinclozolin & 0.51474 \\
$\beta$-Tubulin codon 200 & 0.10760 \\
$\beta$-Tubulin codon 198 & $<0.000001^{*}$ \\
Fenpropimorph & $0.00072^{*}$
\end{tabular}

z The $P$ value indicates the probability that the difference in allelic frequency between the populations from the different plant hosts occurred only by chance. $*=$ Probability is significant.

TABLE 6. Number of strains of Botrytis cinerea collected from each different plant species and percentage of strains that belong to the species transposa, considering only transposa and vacuma strains

\begin{tabular}{lcc}
\hline Plant & No. of strains & Percentage transposa \\
\hline Bindwind & 4 & 100 \\
Sheep sorrel & 3 & 33 \\
Scarlet pimpernel & 2 & 100 \\
Ash & 3 & 100 \\
Herb Robert & 8 & 100 \\
Common comphrey & 3 & 33 \\
Plane tree & 3 & 33 \\
Bramble & 17 & 59 \\
Lime tree & 5 & 60 \\
Strawberry & 1 & 0 \\
Cornel tree & 4 & 100 \\
Coronilla & 1 & 100 \\
Milkweed & 5 & 60 \\
Bazel tree & 2 & 100 \\
Common elder & 5 & 80 \\
White clover & 4 & 25 \\
Green peas & 13 & 23 \\
Rapeseed & 4 & 0 \\
Wild rose & 15 & 40 \\
Willow herb & 3 & 0 \\
Yellow weed & & 66 \\
\hline
\end{tabular}


allele frequencies (10), the fact that the alleles of the markers other than Boty and Flipper have different frequencies on different plants could be attributable to different prevalences of transposa and vacuma on these plants.

Both transposa and vacuma strains were found on disease lesions on living plants and on asymptomatic tissues (Table 1). But vacuma strains were more often on asymptomatic tissues than on disease lesions, whereas transposa strains were more often on disease lesions than on asymptomatic tissues. A genetic differentiation test showed that these differences were significant $(P=0.01)$.

Genetic diversity. Another important finding is the high degree of genetic diversity. The 18 markers distinguished 74 different haplotypes in the 107 strains, the most common being found in only $8 \%$ of the strains. Several alleles were detected that had not been detected in strains from grapes, and several alleles from the grape populations were not present in these strains. Nei's mean gene diversity index, which measures the number of alleles and their evenness, was $\mathrm{H}_{\mathrm{s}}=0.24$ for transposa and $\mathrm{H}_{\mathrm{s}}=0.32$ for vacuma. These values are close to those calculated for the grape population for each species $\left(\mathrm{H}_{\mathrm{s}}=0.32\right.$ for vacuma and $\mathrm{H}_{\mathrm{s}}=0.23$ for transposa) (10). As in the previous study (10), vacuma exhibited greater diversity than did transposa.

\section{Carbendazim and Diethofencarb}
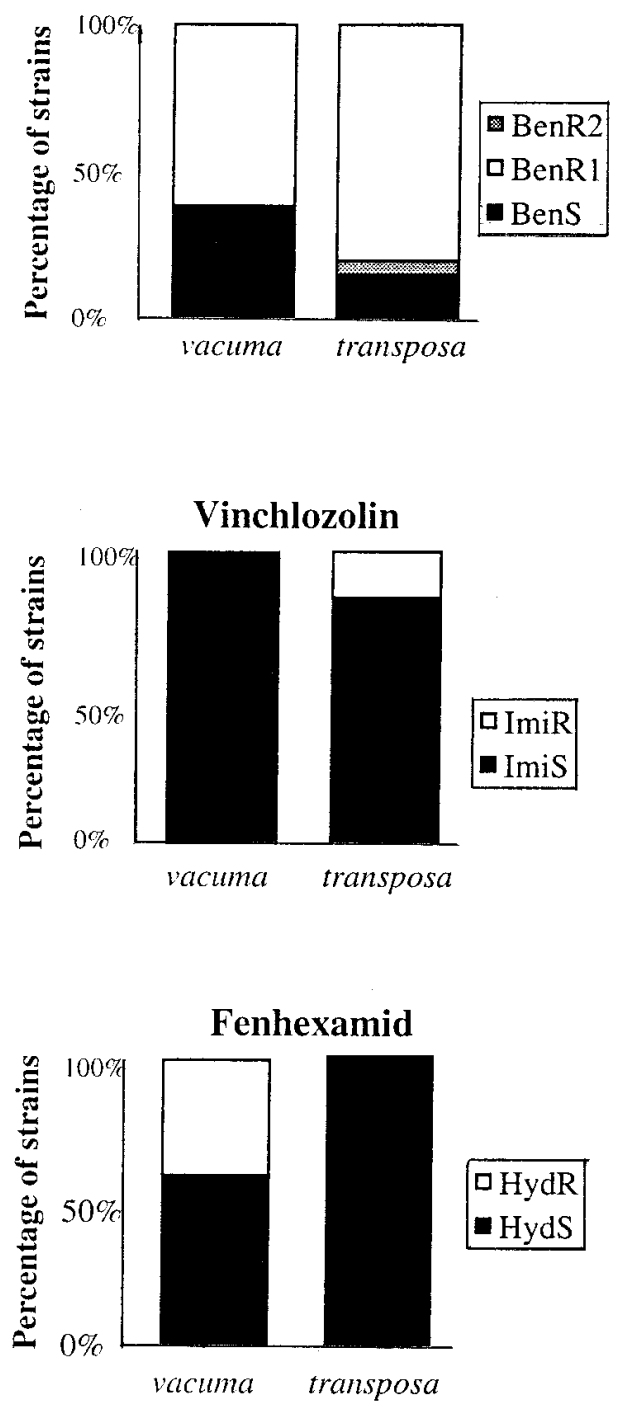

Fig. 2. Frequencies of resistance to various fungicides in strains of the two sibling species of Botrytis cinerea, vacuma and transposa, collected on 21 different plants near grapes in the Champagne region (France).
Since the number of strains collected from each plant was not sufficient to test for gametic disequilibria, we used the genotypic diversity $\mathrm{G}_{\max }(100 \times$ number of haplotypes/number of strains $)$ as an indicator of the occurrence of recombination (3). Genotypic diversity values were $55 \%$ for transposa and $57 \%$ for vacuma. These values are higher than those in asexual populations (3) and are close to those calculated for strains collected from grapes in Champagne (56\% for transposa and $70 \%$ for vacuma).

Fungicide resistances. Figure 2 shows the percentages of fungicide resistance. As observed previously for the strains collected from grapes in June 1994 and June 1995 (10), (i) the BenR1 strains were predominant in both species; (ii) the BenR2 and ImiR phenotypes were present in transposa, but absent in vacuma; and (iii) only vacuma strains were resistant to fenhexamid.

\section{DISCUSSION}

The great genetic diversity detected in this study mirrors that found on grapes (10), in which the diversity was shown to result from both genetic recombination and population subdivision. We did not have enough individuals from each plant to test for gametic disequilibrium, but genetic diversity and genotypic diversity were as high as those on grapes. Gametic disequilibrium studies of the Champagne grape populations demonstrated that genetic recombination occurs, and our findings suggest that sexual reproduction also occurs frequently in this population.

This study shows that the two sibling species of $B$. cinerea identified on grapes also occur elsewhere. Therefore, $B$. cinerea is a complex of at least two species that are sympatric on many plants. However, we found few strains having only the transposable element Boty. Such strains had not been found before in France (10), but some have been identified in Chile (K. LoBuglio, unpublished data). More studies are needed to determine if this means that Boty is invading the species vacuma or if these strains belong to another population.

The genetic differentiation test shows that the frequencies of the markers Flipper and Boty are significantly different on different host plants. The species transposa and vacuma are differentiated by the presence of these transposable elements, such that, even if both occur on the same plants, their relative frequencies are significantly different. For example, vacuma is prevalent on green peas, as observed in 1994 (6), whereas transposa is prevalent on bramble and herb Robert (Table 6). This reinforces the idea that transposa and vacuma do not have the same ability to infect different hosts.

The frequencies of fungicide resistances were close to those found on grapes in June 1994 and June 1995 (10) and provided further evidence of this specialization (Fig. 2). The presence of BenR2 and ImiR phenotypes in transposa can be related to the intensive use of the corresponding fungicides in vineyards. Their absence in vacuma strains confirms that this species is not the major problem on grapes. BenR1 is present in transposa and vacuma, and this can be explained by the fact that carbendazim has long been used alone to spray on grapes and on several other crops. Resistance to fenhexamid, a fungicide that have never been used against grey mold or on any other crop, occurred only in vacuma strains. This resistance is related to increased detoxification of this hydroxyanilide, but this does not confer any direct advantage, because this fungicide is not used in vineyards. The specialization of transposa and vacuma is not yet understood, but there is undoubtedly a difference between the two species in their ability to infect certain plants.

The total percentage of vacuma was similar to those found on grapes in June 1994 and June 1995 (10). The study on grapes suggested a succession of different populations from June to October in Champagne; vacuma was more frequent in June and then decreased until October, when transposa was prevalent. The strains analyzed in this study were collected in June 1997 and they are more like the populations found on grapes in June 1994 and June 1995 than those isolated later in 1994 and 1995 from grapes. This 
suggests that the same succession of populations may occur every year in Champagne.

vacuma Strains were more frequently found on asymptomatic tissues, whereas transposa strains were more frequent on disease lesions. This may reflect different behaviors of the two species, vacuma being more saprophytic and transposa being more like a parasite. It would be consistent with the observed succession of populations: transposa infecting first grapes as a parasite, followed by vacuma acting more saprophytically.

In conclusion, $B$. cinerea is not an unstructured clonal population devoid of specialization, as previously thought. On the contrary, $B$. cinerea undergoes regular sexual reproduction, and it comprises at least two sibling sympatric species, each of which probably has a different ecological niche. This study shows that the population genetics approach can give evidence for population structures and specializations that laboratory tests and field observations failed to detect. But pathogenicity tests are now necessary to confirm and to elucidate the nature of the specialization of the complex $B$. cinerea.

\section{ACKNOWLEDGMENTS}

This work was supported by grants from the Institut National de la Recherche Agronomique, Paris, France. We thank the Comité Interprofessionnel du Vin de Champagne for helping in collecting the samples of Botrytis cinerea, O. Parkes for correcting the English text, and J.-M. Pradier for his valuable comments on the manuscript. We also thank J. W. Taylor for allowing SWAPP to be done in his laboratory and for his valuable comments on the manuscript.

\section{LITERATURE CITED}

1. Armstrong, G., and Armstrong, J. K. 1981. Formae speciales and races of Fusarium oxysporum causing wilt diseases. Pages 391-399 in: Fusarium, Diseases, Biology and Taxonomy. P. E. Nelson, T. A. Tousson, and R. J. Cook, eds. The Pennsylvania State University Press, University Park.

2. Brasier, C. M. 1986. Dynamics of fungal speciation. Pages 215-260 in: Evolutionary Biology of the Fungi. A. D. M. Rayner, C. M. Brasier, and D. Moore, eds. Cambridge University Press, Cambridge.

3. Brygoo, Y., Caffier, V., Carlier, J., Fabre, J.-V., Fernandez, D., Giraud, T., Mourichon, X., Neema, C., Notteghem, J.-L., Pope, C., Tharreau, D., and Lebrun, M.-H. 1998. Reproduction and population structure in phytopathogenic fungi. Pages 133-148 in: Molecular Variability of Fungal Pathogens. P. Bridge, Y. Couteaudier, and J. M. Clarkson, eds. CAB International, Wallingford, United Kingdom.

4. Burt, A., Carter, D. A., White, T. J., and Taylor, J. W. 1994. DNA sequencing with arbitrary primer pairs. Mol. Ecol. 3:523-525.

5. Burt, A., Deidre, A. C., Koenig, G. L., White, T. J., and Taylor, J. W. 1996. Molecular markers reveal cryptic sex in the human pathogen Coccidioides immitis. Proc. Natl. Acad. Sci. U.S.A. 93:770-773.

6. Diolez, A., Marches, F., Fortini, D., and Brygoo, Y. 1994. Boty, a longterminal repeat retroelement in the phytopathogenic fungus Botrytis cinerea. Appl. Environ. Microbiol. 61:103-108.

7. Faretra, F., and Pollastro, S. 1991. Genetic basis of resistance to benzimidazole and dicarboximide fungicides in Botrytis cinerea (Botryotinia fuckeliana). Mycol. Res. 95:943-951.

8. Felsenstein, J. 1981. Skepticism towards Santa Rosalia, or why are there so few kinds of animals? Evolution 35:124-138.

9. Futuyama, D. J., and Moreno, G. 1988. The evolution of ecological specialization. Annu. Rev. Ecol. Syst. 19:207-233.

10. Giraud, T., Fortini, D., Levis, C., Leroux, P., and Brygoo, Y. 1997. RFLP markers show genetic recombination in Botryotinia fuckeliana (Botrytis cinerea) and transposable elements reveal two sympatric species. Mol. Biol. Evol. 14:1177-1185.

11. Giraud, T., Levis, C., Fortini, D., and Brygoo, Y. 1998. The minisatellite MSB1 in the fungus Botrytis cinerea, probably mutates by slippage. Mol. Biol. Evol. 15:1524-1531.

12. Giraud, T., Levis, C., Fortini, D., Leroux, P., and Brygoo, Y. 1998. Plusieurs espèces cachées sous le nom de Botrytis cinerea! Phytoma Déf. Vég. 504:56-60.

13. Green, G. J. 1971. Hybridization between Puccinia graminis tritici and Puccinia graminis secalis and its evolutionary implications. Can. J. Bot. 49:2089-2095.

14. Hiura, U. 1962. Hybridization between varieties of Erysiphe graminis. Phytopathology 52:664-666.

15. Hiura, U. 1978. Genetic basis of some formae speciales in Erysiphe graminis. Page 101 in: The Powdery Mildews. D. M. Spencer, ed. Academic Press, London.

16. Leroux, P., Chapeland, F., Giraud, T., Brygoo, Y., and Gredt, M. 1998. Resistance to sterol biosynthesis inhibitors and various other fungicides in Botrytis cinerea. Pages 297-303 in: Modern Fungicides and Antifungal Compounds. A. Cook, ed. Intercept Ltd., Andover, Hampshire, United Kingdom.

17. Leroux, P., and Clerjeau, M. 1985. Resistance of Botrytis cinerea and Plasmopora viticola in French vineyards. Crop Prot. 4:137-160.

18. Levis, C., Fortini, D., and Brygoo, Y. 1997. Flipper, a mobile Fot1-like transposable element in Botrytis cinerea. Mol. Gen. Genet. 254:674-680.

19. MacFarlane, H. H. 1968. Plant host-pathogen index to volumes 1-40 (1922-1961) of Review of Applied Mycology. Commonwealth Mycological Institute, Kew, England.

20. Maynard Smith, J. 1966. Sympatric speciation. Am. Nat. 100:637-650.

21. Möller, E. M., Bahnweg, G., Sandermann, H., and Geige, H. H. 1992. A simple and efficient protocol for isolation of high molecular weight DNA from filamentous fungi, fruit bodies, and infected plant tissues. Nucleic Acids Res. 20:6115-6116.

22. Nei, M. 1973. Analysis of gene diversity in subdivided populations. Proc. Natl. Acad. Sci. U.S.A. 70:3321-3323.

23. Raymond, M. 1995. A population genetics software for exact tests and eucumenicism. J. Hered. 86:248-249.

24. Rice, W. R. 1987. Selection via habitat specialization: The evolution of reproductive isolation as a correlated character. Evol. Ecol. 1:301-314.

25. Savile, D. B. 1971. Coevolution of the rust fungi and their hosts. Q. Rev. Biol. 46:211.

26. Suty, A., Pontzen, R., and Stenzel, K. 1997. KBR 2738 mode d'action et sensibilité de Botrytis cinerea. Pages 561-568 in: $5^{\circ}$ Conférence internationale sur les maladies des plantes. Association Nationale de Protection des Plantes (ANPP), Paris.

27. Urban, Z. 1980. Rust ecology and phytocenology as aids in rust taxonomy. Rep. Tottori Mycol. Inst. 18:269.

28. Yarden, O., and Katan, T. 1993. Mutations leading to substitutions at amino acids 198 and 200 of beta-tubulin that correlate with benomyl-resistance phenotypes of field strains of Botrytis cinerea. Phytopathology 83:1478-1483. 